\title{
Produtividade e massa fresca de bulbos de cebola sob densidades de plantio no Vale do São Francisco
}

\author{
Geraldo M de Resende; Nivaldo Duarte Costa \\ Embrapa Semi-Árido, C. Postal 23, 56300-970 Petrolina-PE; E-mail: gmilanez@cpatsa.embrapa.br
}

\begin{abstract}
RESUMO
Com o objetivo de avaliar o efeito de diferentes espaçamentos entre as linhas e entre as plantas sobre as características produtivas e a conservação pós-colheita dos bulbos de cebola, conduziu-se um experimento no período de setembro de 1999 a março de 2000, no Campo Experimental de Bebedouro, da Embrapa Semi-Árido, em Petrolina-PE. O delineamento experimental utilizado foi de blocos ao acaso, no esquema fatorial $2 \times 3$, compreendendo dois espaçamentos entre as linhas $(0,10$ e $0,15 \mathrm{~m})$ e três espaçamentos entre as plantas $(0,10,0,20$ e $0,30 \mathrm{~m})$, sendo utilizada a cultivar Texas Grano 502 PRR com quatro repetições. Para produtividade comercial verificou-se que o espaçamento de $0,10 \mathrm{~m}$ entre as linhas apresentou maior produtividade $\left(37,8 \mathrm{t} \mathrm{ha}^{-1}\right)$ comparativamente ao espaçamento de $0,15 \mathrm{~m}\left(34,4 \mathrm{t} \mathrm{ha}^{-1}\right)$, ocorrendo reduções lineares com o aumento do espaçamento entre as plantas. À medida que se aumentou o espaçamento entre as linhas e as plantas observou-se redução gradativa na produção de bulbos não comerciais. A massa fresca dos bulbos aumentou linearmente à medida que se aumentaram os espaçamentos entre as linhas e as plantas. Uma maior porcentagem de bulbos pequenos e médios foi verificada nos menores espaçamentos. Â medida que se incrementou o espaçamento entre as plantas ocorreu uma maior perda de massa dos bulbos de cebola.
\end{abstract}

Palavras-chave: Allium cepa L., rendimento, espaçamento, conservação pós-colheita.

\begin{abstract}
Yield characteristics and fresh mass of onion under planting densities in the São Francisco Valley

With the objective of evaluating the effect of different planting spacings on yield characteristics and bulb weight loss, an experiment was carried out from September 1999 to March 2000, in Petrolina, Pernambuco State, Brazil, in a randomized complete block design, in a $2 \times 3$ factorial scheme, with four replications. The cultivar Texas Grano 502 PRR was planted at 0.10 and $0.15 \mathrm{~m}$ row spacings and at $0.10 ; 0.15$ and $0.30 \mathrm{~m}$ plant spacings. For commercial yield, it was verified that the row spacing of $0,10 \mathrm{~m}$ showed highest yield $(37.8 \mathrm{t}$

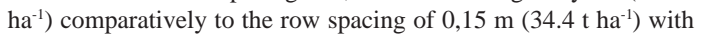
linear reductions with the increase in plant spacing. The fresh mass of bulbs increased linearly as the spacings between rows and between plants increased. Larger percentages of small and medium bulbs were obtained with the narrowest spacings. As spacing between plants increased, a larger loss of onion bulb mass was found.
\end{abstract}

Keywords: Allium cepa L., yield, spacing, postharvest conservation.

\section{(Recebido para publicação em 19 de setembro de 2005; aceito em 3 de abril de 2006)}

A produção mundial de cebola (Allium cepa L.), em 2004, foi de 53,6 milhões de toneladas, cultivadas em uma área 3,1 milhões de hectares, o que proporcionou uma produtividade média de 17,5 t/ha (FAO, 2005). No Brasil, a cebola ocupa o terceiro lugar em importância econômica (Souza \& Resende, 2002). A produtividade média nacional se situou em 17,9 t/ha, sendo que nos estados de Pernambuco e Bahia, maiores produtores do Nordeste, se alcançou uma produtividade média de 21,2 e 24,2 t/ha, respectivamente (IBGE, 2005).

Estudando os espaçamentos de 0,10 x $0,15 \mathrm{~m}$ e $0,20 \times 0,15 \mathrm{~m}$, Viegas D'Abreu (1996), verificou que a maior produtividade foi obtida no menor espaçamento $(0,10 \times 015 \mathrm{~m})$, e que a maior massa fresca do bulbo $(145,7 \mathrm{~g}$. bulbo $^{-1}$ ) foi verificada no maior espaçamento comparado aos $118,0 \mathrm{~g}$. bulbo $^{-1}$, obtidos com $0,10 \times 0,15 \mathrm{~m}$.
Avaliando a influência da densidade de plantas sobre a produtividade em três espaçamentos $(0,20 ; 0,30$ e $0,40 \mathrm{~m}$ x $0,08 \mathrm{~m}$ ), Lopes et al. (2004) constataram que no menor espaçamento $(0,20 \mathrm{x}$ $0,08 \mathrm{~m}$ ) foram obtidas a maior produtividade comercial e menor massa fresca do bulbo. Kanton et al. (2002) observaram aumento na produtividade com o incremento da densidade de plantio (37,04 para 156,25 plantas por metro quadrado), assim como menor altura de planta e massa fresca do bulbo, relatando que densidades acima de 76,92 plantas $\mathrm{m}^{-2}$ como aquelas que proporcionam maiores produtividades de bulbos comerciais. Dellacecca \& Lovato (2000), relataram resultados similares onde a densidade de 80 plantas por metro quadrado obteve a maior produtividade e menor massa fresca do bulbo comparativamente a 26,6 e 40,0 plantas $\mathrm{m}^{-2}$.
O aumento do espaçamento de plantio de 0,05 para $0,15 \mathrm{~m}$ entre plantas proporcionando redução na produtividade total e comercial e na massa fresca do bulbo é também relatado por Santos et al. (2000) para a cultivar Texas Grano 502. Por outro lado, avaliando a mesma cultivar, Sabota \& Downes (1981) não observaram diferenças significativas de produtividade quando compararam duas populações de plantas (192.940 e 257.320 plantas. ha ${ }^{-1}$ ).

A cebola é uma planta de dias longos quanto à formação de bulbos, e as cultivares designadas de dias curtos não são, particularmente, plantas de dias curtos; simplesmente exigem menos horas de luz para bulbificarem (Melo \& Ribeiro, 1990). A formação de bulbos está relacionada com a interação entre a temperatura e o fotoperíodo. Nesta interação o fator mais importante é o 
fotoperíodo e o mesmo determina os limites de adaptação das diferentes cultivares (Galmarini, 1997). A região Nordeste, representada pelos estados de Pernambuco e Bahia, privilegiada pelas suas condições climáticas, no caso do cultivo da cebola, pratica a semeadura de janeiro a dezembro, com concentração nos meses de janeiro a março, possibilitando um escalonamento de plantio e produção com oferta em diferentes períodos (Costa et al., 2002).

Pelo exposto, assim como pela carência de informações técnicas sobre o cultivo da cebola no período de verão, o presente estudo objetivou avaliar o efeito de diferentes densidades de plantio sobre as características produtivas e a conservação pós-colheita dos bulbos de cebola Texas Grano 502 PRR, nas condições do Vale do São Francisco.

\section{MATERIAL E MÉTODOS}

O experimento foi conduzido no período de setembro de 1999 a março de 2000, em campo experimental da Embrapa Semi-Árido, em Petrolina-PE. O solo classificado como Latossolo Vermelho Amarelo Distrófico, apresentou $\mathrm{pH}\left(\mathrm{H}_{2} \mathrm{O}\right)=6,1 ; \mathrm{Ca}=2,1 \mathrm{cmol}_{\mathrm{c}} \mathrm{dm}^{-3}$; $\mathrm{Mg}=0,7 \mathrm{cmol}_{\mathrm{c}} \mathrm{dm}^{-3} ; \mathrm{Na}=0,01 \mathrm{cmol}_{\mathrm{c}}$ $\mathrm{dm}^{-3}, \mathrm{~K}=0,28^{\mathrm{c}} \mathrm{cmol}_{\mathrm{c}} \mathrm{dm}^{-3} ; \mathrm{Al}=0,05^{\mathrm{c}}$ $\mathrm{cmol}_{\mathrm{c}} \mathrm{dm}^{-3}, \mathrm{P}($ Mehlich $)=7,2 \mathrm{mg} \mathrm{dm}^{-3} \mathrm{e}$ M.O. $=6,9$ g. $\mathrm{kg}^{-1}$.

$\mathrm{O}$ delineamento experimental utilizado foi blocos ao acaso, em esquema fatorial $2 \times 3$, compreendendo dois espaçamentos entre as linhas $(0,10 \mathrm{e}$ $0,15 \mathrm{~m})$ e três espaçamentos entre as plantas $(0,10 ; 0,20$ e $0,30 \mathrm{~m})$ com quatro repetições. A unidade experimental constituiu-se de um canteiro de $3,0 \mathrm{~m}$ de comprimento por 1,2 $\mathrm{m}$ de largura, sendo usado como área útil 1,8 m² (3,0 x 0,6 m). A adubação de plantio constou de $600 \mathrm{~kg}$. ha- ${ }^{-1}$ da fórmula NPK 624-12, baseada na análise do solo. Foram aplicados em cobertura $90 \mathrm{~kg}$. ha- ${ }^{-1}$ de $\mathrm{N}$ na forma de uréia e $30 \mathrm{~kg}$. ha ${ }^{-1}$ de $\mathrm{K}_{2} \mathrm{O}$ na forma de cloreto de potássio, parcelados aos 15 e 30 dias após o transplantio.

Foi utilizada a cultivar Texas Grano 502 PRR, sendo a semeadura feita em 14 de setembro de 1999 e o transplantio efetuado 25 dias após.

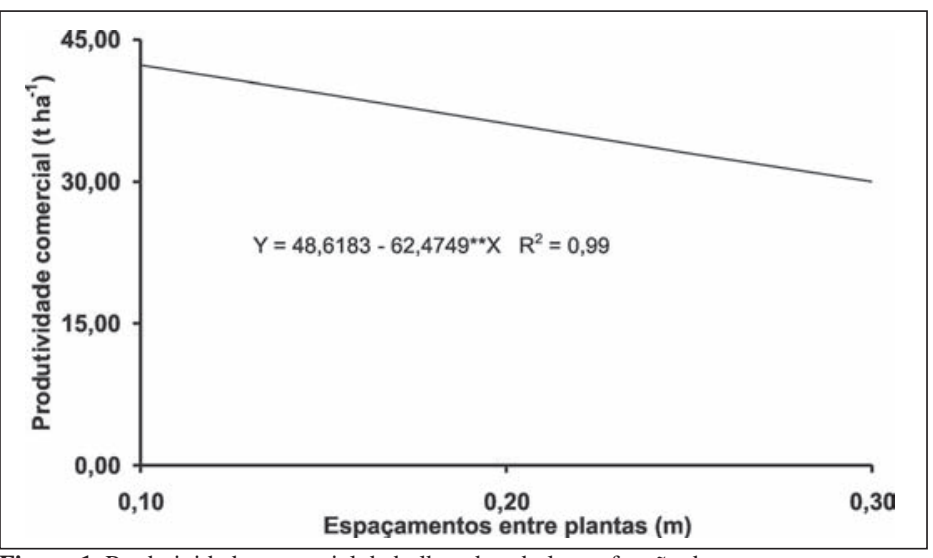

Figura 1. Produtividade comercial de bulbos de cebola em função dos espaçamentos entre as plantas. Petrolina, Embrapa Semi-Árido, 1999/2000.

O preparo do solo constou de aração, gradagem e levantamento dos canteiros a $0,20 \mathrm{~m}$ de altura. A cultura foi mantida no limpo através de capinas manuais. A irrigação por microaspersão foi realizada três vezes por semana, com lâminas em torno de $10 \mathrm{~mm}$, baseada na evaporação do tanque classe $\mathrm{A}$, e suspensas 20 dias antes da colheita. Os demais tratos fitossanitários foram realizados de acordo com as recomendações para a cultura da cebola.

A colheita foi realizada em 22 de dezembro de 1999 quando as plantas apresentaram sinais avançados de senescência, como amarelecimento e seca das folhas e quando mais de $70 \%$ das plantas encontravam-se estaladas. A cura foi realizada ao sol por três dias e 12 dias à sombra em galpão ventilado.

Foram avaliadas a produtividade comercial de bulbos (bulbos perfeitos e com diâmetro transversal acima de 35 $\mathrm{mm}$ ) e refugos (bulbos com diâmetro inferior a $35 \mathrm{~mm}$ ) expressos em $\mathrm{t} \mathrm{ha}^{-1}$, aos 15 dias após a cura. A massa fresca de bulbo (g. bulbo ${ }^{-1}$ ) foi determinada dividindo-se a massa de bulbos comerciais após a cura pelo número de bulbos. Após o período de cura, os bulbos foram armazenados à temperatura ambiente e realizadas pesagens aos 20,40 e 60 dias, sendo os valores comparados àqueles obtidos ao final da cura (15 dias após colheita). Os valores foram transformados em porcentagem de perda de massa. A classificação de bulbos comerciais segundo o diâmetro transversal (mm) foi feita de acordo com Brasil (1995) em Classe 2: maior que 35 até 50 mm de diâmetro; Classe 3: maior que 50 até $70 \mathrm{~mm}$; Classe 4: maior que 70 até $90 \mathrm{~mm}$ e Classe 5: maior que $90 \mathrm{~mm}$. Os dados foram expressos em porcentagem.

Segundo a metodologia descrita por Pimentel Gomes (2000), os dados coletados foram submetidos à análise de variância e as médias comparadas pelo teste de Tukey, ao nível de 5\% de probabilidade e regressão polinomial. Os dados de porcentagem foram transformados em arco-seno $\sqrt{P / 100}$ para efeitos de análise, sendo apresentados nos resultados as médias originais.

\section{RESULTADOS E DISCUSSÃO}

Houve efeitos significativos dos espaçamentos entre as linhas e entre as plantas de forma independente em relação a produtividade comercial, não ocorrendo interação entre esses fatores. No espaçamento de $0,10 \mathrm{~m}$ entre as linhas a produtividade foi superior $\left(37,8 \mathrm{t} \mathrm{ha}^{-1}\right)$ comparativamente ao espaçamento de $0,15 \mathrm{~m}\left(34,4 \mathrm{tha}^{-1}\right)$. Verificou-se reduções lineares com o aumento do espaçamento entre as plantas (Figura 1), tendo obtido os maiores rendimentos $\left(43,4 \mathrm{t} \mathrm{ha}^{-1}\right)$ no espaçamento de $0,10 \mathrm{~m}$ entre as plantas. Estes resultados confirmam as observações de que as cebolas respondem especialmente a variação no espaçamento entre as plantas 


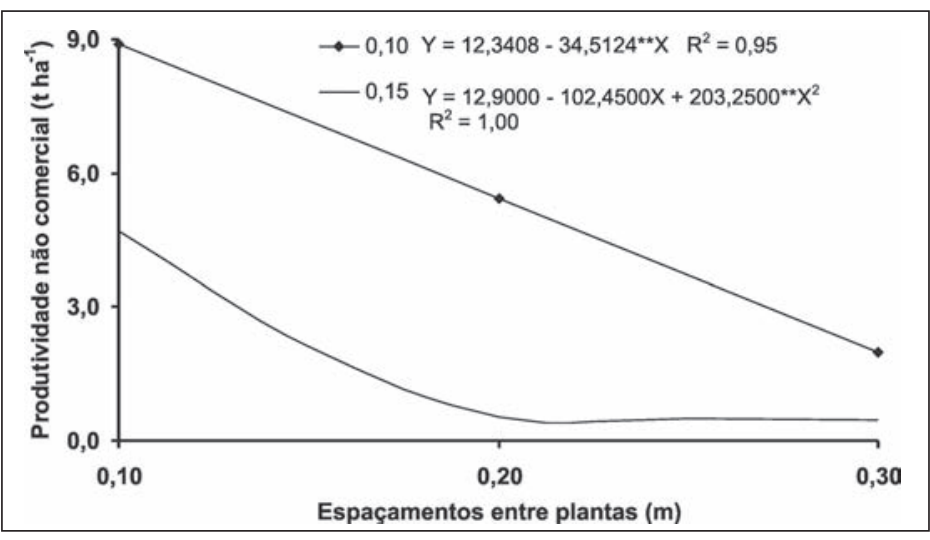

Figura 2. Refugos (produtividade não comercial) de bulbos de cebola nos espaçamentos 0,10 e 0,15 m entre as linhas em função dos espaçamentos entre as plantas. Petrolina, Embrapa Semi-Árido, 1999/2000.

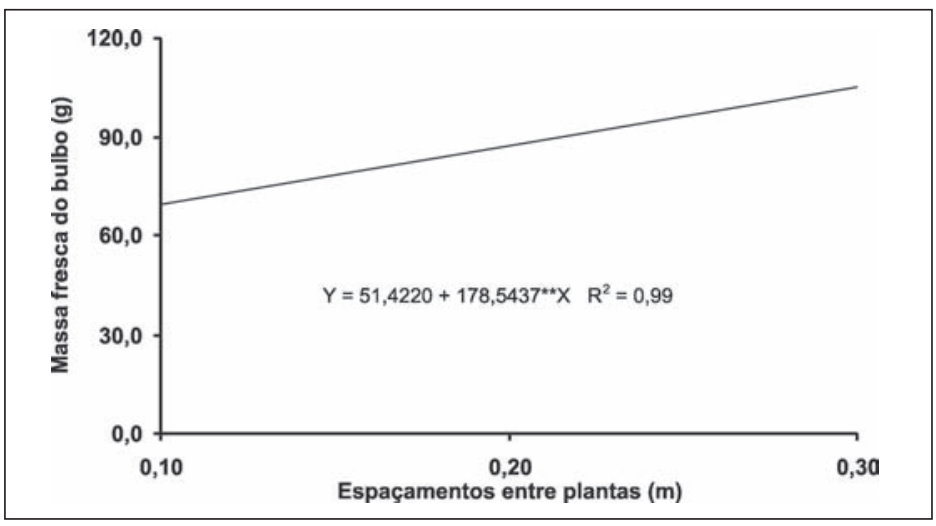

Figura 3. Massa fresca do bulbo de cebola em função dos espaçamentos entre as plantas. Petrolina, Embrapa Semi-Árido, 1999/2000.

(Filgueira, 1982; Melo et al., 1988). Entretanto, a produtividade da cebola aumentou à medida que se diminuiu o espaçamento entre as linhas e entre as plantas, fato relatado por diversos autores em diferentes épocas (Stoffella, 1996; Boff et al., 1998; Santos et al.; 2000).

Bulbos charutos, podres, ou com danos mecânicos não foram observados no que se refere à produção de refugos (bulbos não comerciais), havendo somente bulbos com diâmetro inferior a $35 \mathrm{~mm}$. Para esta característica obtevese efeitos significativos da interação verificando-se uma redução gradativa na produção de bulbos não comerciais no espaçamento de $0,10 \mathrm{~m}$ entre as linhas à medida que se aumentou o espaçamento entre as plantas (Figura 2). Pela derivada da equação de regressão constatou-se que o menor rendimento de bulbos não comerciais foi obtido no espaçamento de $0,25 \mathrm{~m}$ entre as plantas, para o espaçamento de 0,15 m entre as linhas (Figura 2). Estes resultados mostram relação inversa do espaçamento entre as linhas e entre as plantas e produtividade de bulbos de cebola. Em populações menores se produz, geralmente, baixos rendimentos e alta porcentagem de bulbos médios e grandes. Em cultivos com densidades maiores que a ótima, se têm bulbos pequenos e desuniformes de qualidade comercial inferior, comparativamente ao cultivo em densidade adequada.
Não se observou efeito significativo da interação espaçamento entre as linhas e entre as plantas para massa fresca do bulbo. No espaçamento de $0,15 \mathrm{~m}$ entre as linhas se obteve maior massa fresca $\left(95,52 \mathrm{~g}\right.$. bulbo $\left.{ }^{-1}\right)$ comparativamente ao espaçamento de $0,10 \mathrm{~m}(78,73$ g. bulbo $\left.{ }^{-1}\right)$. O coeficiente angular da equação de regressão indica ser este incremento da ordem de 17,86 g. bulbo ${ }^{-1}$ para cada aumento do espaçamento entre as plantas (Figura 3). Salienta-se que a maior massa fresca do bulbo foi obtida tanto com o aumento do espaçamento entre as plantas como entre as linhas, o que, provavelmente, está relacionado à maior área de exploração das raízes e menor competição pelos fatores de produção como água, luz e nutrientes. Lopes (1987) constatou para diferentes cultivares que com o incremento da densidade ocorreu redução no diâmetro e na massa fresca do bulbo. A diminuição da massa fresca do bulbo com o incremento da densidade de plantio foi também observado por outros autores (Galmarini \& Gaspera, 1995; Stoffella, 1996; Lipinski et al., 2002).

A análise de variância para classificação de bulbos de cebola em porcentagem, em classes, segundo o diâmetro transversal indica efeito significativo da interação entre os fatores estudados para todas as classes (Tabela 1). No que se refere à classificação de bulbos classe 2 , constatou-se para os espaçamentos de 0,10 e $0,15 \mathrm{~m}$ entre as linhas reduções lineares com o aumento do espaçamento entre as plantas. À medida que se aumentou o espaçamento, foi observada redução gradativa na porcentagem deste tipo de bulbo, que são menores comparativamente às demais classes. No entanto, no que se refere à classe 3 (bulbos de tamanho intermediário), ocorreram aumentos lineares com o incremento dos espaçamentos entre as plantas para o espaçamento de $0,10 \mathrm{~m}$ entre as linhas e efeito quadrático constando-se menor porcentagem desse tipo no espaçamento de $0,22 \mathrm{~m}$ entre as plantas para o espaçamento de $0,15 \mathrm{~m}$ entre as linhas.

Para a classe 4, que são bulbos maiores comparativamente às demais classes, registrou-se um aumento linear à medida que se aumentou o espaçamento 
entre as plantas (Tabela 1). Resultados estes que corroboram os de Viegas D'Abreu (1996), que obteve aumento no tamanho de bulbo no maior espaçamento, assim como os obtidos por Stoffella (1996) que verificou maior porcentagem de bulbos pequenos e médios nos menores espaçamentos, e Rumpel \& Felczynski (2000) que encontraram redução na produção de bulbos maiores com o incremento da densidade de plantio.

Os resultados obtidos no presente trabalho evidenciaram uma relação inversa entre a densidade de plantio e o tamanho do bulbo, onde maiores densidades proporcionaram menores bulbos, e vice-versa. Segundo Souza \& Resende (2002), o mercado consumidor nacional prefere bulbos de tamanho médio com massas de 80 a 100 gramas e diâmetro transversal de 40 a $80 \mathrm{~mm}$. Neste contexto observou-se para o presente estudo variações de 78,73 $(0,10 \mathrm{~m})$ e $95,52 \mathrm{~g}$. bulbo ${ }^{-1}(0,15 \mathrm{~m})$ para o espaçamento entre as linhas e 68,91 $(0,10 \mathrm{~m}), 87,86(0,20 \mathrm{~m})$ e $104,98 \mathrm{~g}$. bulbo $^{-1}(0,30 \mathrm{~m})$ para o espaçamento entre as plantas, valores esses que satisfazem praticamente às exigências de mercado. A perda de massa aos 20, 40 e 60 dias após cura (DAC) foi influenciada significativamente pelo espaçamento entre as linhas e as plantas, assim como pela interação desses fatores (Tabela 2). Para porcentagem de perda de massa aos 20 dias após cura, verificou-se efeitos da interação evidenciando maiores perdas com o incremento dos espaçamentos entre as plantas. Pelos coeficientes angulares das equações de regressão estimou-se esta perda de massa em 1,38 $(0,10 \mathrm{~m})$ e $1,14 \%(0,15 \mathrm{~m})$ para cada aumento do espaçamento entre as plantas (Tabela 2).

Para a perda de massa aos 40 DAC (Tabela 2) obteve-se efeito significativo do espaçamento entre as linhas, no qual o espaçamento de $0,15 \mathrm{~m}$ apresentou maior perda com $11,22 \%$ contra $10,13 \%$ do espaçamento com $0,10 \mathrm{~m}$. Em relação aos espaçamentos entre as plantas obteve-se um efeito linear positivo, ou seja, à medida que se incrementou o espaçamento entre as plantas ocorreu uma maior perda de massa dos bulbos de cebola. Pelo coefi-

Tabela 1. Equações de regressão para classificação de bulbos de cebola, em classes (\%), segundo o diâmetro transversal, nos espaçamentos 0,10 e $0,15 \mathrm{~m}$ entre as linhas em função dos espaçamentos entre as plantas. Petrolina, Embrapa Semi-Árido, 1999/2000.

\begin{tabular}{lll}
\hline Classes & Equações de regres são & \\
\hline \multirow{2}{*}{ Classe 2} & $\mathrm{Y}(0,10 \mathrm{~m})=59,8872-123,9166^{* *} \mathrm{X}$ & $\mathrm{R}^{2}=0,94$ \\
& $\mathrm{Y}(0,15 \mathrm{~m})=52,8103-100,6907^{* *} \mathrm{X}$ & $\mathrm{R}^{2}=0,99$ \\
\hline \multirow{2}{*}{ Classe 3 } & $\mathrm{Y}(0,10 \mathrm{~m})=31,0159+61,4908^{* *} \mathrm{X}$ & $\mathrm{R}^{2}=0,95$ \\
& $\mathrm{Y}(0,15 \mathrm{~m})=20,2927+277,0745 \mathrm{X}-639,8282^{* *} \mathrm{X}^{2}$ & $\mathrm{R}^{2}=1,00$ \\
\hline \multirow{2}{*}{ Classe 4 } & $\mathrm{Y}(0,10 \mathrm{~m})=8,0117+81,6439^{* *} \mathrm{X}$ & $\mathrm{R}^{2}=0,89$ \\
& $\mathrm{Y}(0,15 \mathrm{~m})=7,1950+87,9335{ }^{* *} \mathrm{X}$ & $\mathrm{R}^{2}=0,91$ \\
\hline
\end{tabular}

** Significativo ao nível de $1 \%$ de probabilidade, pelo teste de $\mathrm{F}$.

Tabela 2. Equações de regressão para porcentagem de perda de massa aos 20 , 40 e 60 dias após a cura (DAC) de bulbos de cebola em função dos espaçamentos entre as linhas e entre as plantas. Petrolina, Embrapa Semi-Árido, 1999/2000.

\begin{tabular}{lll}
\hline Características & Equações de regressão & \\
\hline \multirow{2}{*}{$20 \mathrm{DAC}$} & $\mathrm{Y}(0,10 \mathrm{~m})=11,1278+12,8397^{* *} \mathrm{X}$ & $\mathrm{R}^{2}=0,95$ \\
& $\mathrm{Y}(0,15 \mathrm{~m})=11,1084+11,3773^{* *} \mathrm{X}$ & $\mathrm{R}^{2}=0,91$ \\
\hline $40 \mathrm{DAC}$ & $\mathrm{Y}=13,3614+28,5261^{* *} \mathrm{X}$ & $\mathrm{R}^{2}=0,97$ \\
\hline $60 \mathrm{DAC}$ & $\mathrm{Y}=16,7703+31,0200^{* *} \mathrm{X}$ & $\mathrm{R}^{2}=0,92$ \\
\hline
\end{tabular}

** Significativo ao nível de $1 \%$ de probabilidade, pelo teste de F.

ciente angular da equação de regressão esta perda foi estimada em $2,85 \%$ para cada aumento no espaçamento. Resultados similares foram observados quanto à perda de massa aos $60 \mathrm{DAC}$, onde o espaçamento entre as plantas de 0,15 $\mathrm{m}$ resultou em maior perda com $15,91 \%$ contra $14,56 \%$ do espaçamento com $0,10 \mathrm{~m}$. No que se refere ao espaçamento entre as plantas constatou-se um efeito linear positivo, sendo a perda estimada em $3,10 \%$, para cada aumento no espaçamento (Tabela 2). Inferiu-se por estes resultados, que a maior perda ocorreu em espaçamentos mais largos, que determinaram bulbos maiores, com conseqüente teores de água mais elevados, no entanto, de pequena magnitude. Resultados similares foram observados por Trevisan et al. (1999) que encontraram após 100 dias de armazenamento, em condições naturais, $15 \%$ de perda de massa de bulbos para diferentes cultivares estudadas. Já Calbo et al. (1980) observaram perdas de $30 \%$ a $100 \%$ em bulbos de cebola "Baia Periforme" armazenadas até 70 dias, a granel e réstias, em diferentes recipientes.

Em função dos resultados obtidos, recomenda-se os espaçamentos de 0,10 e $0,15 \mathrm{~m}$ entre as linhas e $0,10 \mathrm{~m}$ entre as plantas como os mais adequados para o cultivo da cebola cultivar Texas Grano
502 PRR, nas condições do Vale do São Francisco, para plantio no segundo semestre do ano (verão).

\section{REFERÊNCIAS}

BOFF P; HENRI S; GONÇALVES PAS. 1998. Influência da densidade de plantas na ocorrência de doenças foliares e produção de bulbos de cebola. Fitopatologia Brasileira 23: 448452.

BRASIL. Ministério da Agricultura, Abastecimento e Reforma Agrária. 1995. Portaria n.529 de 18 ago. 1995. Diário Oficial da República Federativa do Brasil, Brasília: p.13513.

CALBO AG; CORDEIRO CMT; GUALBERTO AG. 1980. Efeito do acondicionamento sobre o comportamento fisiológico e conservação de duas cultivares de cebola (Allium cepa) no Vale do São Francisco. Revista de Olericultura 18: 86-99.

COSTA ND; LEITE DL; SANTOS CAF; CANDEIA JA; VIDIGAL SM. 2002. Cultivares de cebola. Informe Agropecuário 23: 2027.

DELLACECCA V; LOVATO AFS. 2000. Effects of different plant densities and planting systems on onion (Allium cepa $\mathrm{L}$.) bulb quality and yield. Acta Horticulturae 533: 197-203.

FAO Agricultural production, primary crops. 2005, 26 de janeiro. Disponível em http:// www.fao.org

FILGUEIRA FAR. 1982. Manual de Olericultura: cultura e comercialização de hortaliças. 2. ed. São Paulo: Agronômica Ceres. 357p.

GALMARINI CR. 1997. Caracteristicas botanicas y fisiologicas. In: Manual del cultivo de la cebolla. GALMARINI CR. (eds). San Juan: INTA. p. 128 
GALMARINI CR; GASPERA PG. 1995. Efecto de la epoca de transplante y la densidad de plantacion en el cultivo de cebolla tipo Valenciana. Horticultura Argentina 14: 23-29.

INSTITUTO BRASILEIRO DE GEOGRAFIAE ESTATÍSTICA - IBGE. 2005, 26 de janeiro. Produção Agrícola Municipal. Disponível em http://www.sidra.ibge.gov.br

KANTON RAL; ABBEY L; HILLA RG; TABIL MA; JAN ND. 2002. Density affects plant development and yield of bulb onion (Allium cepa L.) in Northern Ghana. Journal Vegetable Crop Production 8: 5-25.

LIPINSKI VM, GAVIOLA S; GAVIOLA JC. 2002. Efecto de la densidad de plantación sobre el rendimiento de cebolla cv. Cobriza Inta con riego por goteo. Agricultura Técnica 62: 574-582.

LOPES JF. 1987. Effects of planting dates and spacing on several characteristics of short day onion varieties grown in South Texas. Texas: University. 111p (Ph.D. thesis).

LOPES MC; CZEPAK MP; SIRTOLI LF. 2004. Avaliação de diferentes espaçamentos na produtividade de três cultivares de cebola. In: CONGRESSO BRASILEIRO DE OLERICULTURA, 44. Resumos... Campo Grande: SOB (CD-ROM).
MELO PCT; RIBEIRO A. 1990. Produção de sementes de cebola: cultivares de polinização aberta e híbridos. In: CASTELLANE PD; NICOLOSI WM; HASEGAWA M (eds). Produção de sementes de hortaliças. Jaboticabal: FCAV/UNESP. p. 15-59.

MELO PCT; RIBEIRO A; CHURATA-MASCA MGC. 1988. Sistemas de produção, cultivares de cebola e seu desenvolvimento para as condições brasileiras. In: SEMINÁRIO NACIONAL DE CEBOLA, 3. Anais... Jaboticabal: FUNEP. p. 27-61.

PIMENTEL GOMES F. 2000. Curso de estatística experimental. 14. ed. São Paulo: Nobel. 477p.

RUMPEL J; FELCZYNSKI K. 2000. Effect of plant density on yield and bulb size of direct sown onions. Acta Horticulturae 533: 179-186.

SABOTA CM; DOWNES JD. 1981. Onion growth na yield in relation to transplant, prunning, size, spacing and depth of planting. Hortscience 16: 533-535.
SANTOS HS; TANAKA MT; WATANABE SH; ARANTES PAZ; INOUE TT. 2000. Produção de cebola em função de tamanho de muda e espaçamento. In: CONGRESSO BRASILEIRO DE OLERICULTURA, 40. Resumos... SOB (Suplemento).

SOUZA RJ; RESENDE GM. 2002. Cultura da cebola. Lavras: UFLA. 115p. (Textos Acadêmicos - Olericultura, 21).

STOFFELLAPJ. 1996. Planting arrangement and density of transplants influence sweet Spanish onion yields and bulb size. Hortscience 31: 1129-1130.

TREVISAN JN; MARTINS GAK; LOPES SJ; GARCIA DC. 1999. Rendimento e conservabilidade pós-colheita de genótipos de cebola cultivados em solo de várzea. Ciência Rural 29: 409-413.

VIEGAS D'ABREU D A. 1996. Effect of nitrogen and spacing on bulb splitting in onion $\mathrm{cv}$. Bombay Red. TVIS Newsletter 1: 26 